\title{
$\sigma$ meson exchange effect on non-mesonic hypernuclear weak decay observables
}

\author{
C. Barbero* and A. Mariano \\ Departamento de Física, Facultad de Ciencias Exactas, Universidad Nacional de La Plata, C.C. 67, 1900 La Plata, Argentina
}

(Received 13 July 2005; published 13 February 2006)

\begin{abstract}
We analyze the influence of $\sigma$ meson exchange on the main non-mesonic hypernuclear weak decay observables: Total rate $\Gamma_{\mathrm{NM}}$, neutron-to-proton branching ratio $\Gamma_{n / p}$, and proton asymmetry parameter $a_{\Lambda}$. The $\sigma$ meson exchange is added to the standard strangeness-changing weak $\Lambda N \rightarrow N N$ transition potential, which includes the exchange of the complete pseudoscalar and vector mesons octet $\left(\pi, \eta, K, \rho, \omega, K^{*}\right)$. Using a shell model formalism, the $\sigma$ meson weak coupling constants are adjusted to reproduce the recent $\Gamma_{\mathrm{NM}}$ and $\Gamma_{n / p}$ experimental data for ${ }_{\Lambda}^{5} \mathrm{He}$. Numerical results for the remaining observables of ${ }_{\Lambda}^{5} \mathrm{He}$ and all the observables of ${ }_{\Lambda}^{12} \mathrm{C}$ decays are presented. They clearly show that the addition of the $\sigma$ meson improves the agreement with the data. However, its effect it is not enough to reproduce well the $a_{\Lambda}$ values, which means that other improvements of the model must be explored to explain the puzzle posed by the experiments.
\end{abstract}

DOI: 10.1103/PhysRevC.73.024309 PACS number(s): 21.80.+a, 25.80.Pw, 21.60.-n, 13.75.Ev

The free decay of a $\Lambda$ hyperon occurs almost exclusively through the mesonic mode, $\Lambda \rightarrow \pi N$, emerging the nucleon with a momentum of about $100 \mathrm{MeV} / c$. Inside the nuclear medium $\left(p_{F} \sim 270 \mathrm{MeV} / c\right)$, this mode is Pauli blocked; and for all but the lightest $\Lambda$ hypernuclei $(A \geqslant 5)$, the weak decay is dominated by the non-mesonic channel, $\Lambda N \rightarrow N N$, with enough kinetic energy to put the two emitted nucleons above the Fermi surface. The non-mesonic hypernuclear weak decay (NMHWD) offers us a very unique opportunity to investigate the strangeness-changing weak interaction between hadrons. Assuming that the NMHWD is saturated by the $\Lambda N \rightarrow N N$ mode, the transitions receive contributions either from neutrons $(\Lambda n \rightarrow n n)$ and protons $(\Lambda p \rightarrow n p)$, with rates $\Gamma_{n}$ and $\Gamma_{p}$, respectively $\left(\Gamma_{\mathrm{NM}}=\Gamma_{n}+\Gamma_{p}, \Gamma_{n / p}=\Gamma_{n} / \Gamma_{p}\right)$. Over the last three decades, a huge amount of theoretical and experimental effort has been invested in solving an interesting puzzle: The theoretical models cannot reproduce simultaneously the experimental values of $\Gamma_{n / p}$ and $a_{\Lambda}$.

From the experimental side, there is actually an intense activity, as can be seen in the light of the experiments under way and/or planned at (KEK) [1], (FINUDA) [2], and Brookhaven National Laboratory (BNL) [3]. The preliminary results give a $\Gamma_{n / p}$ ratio value very close to 0.5 [4-6], and the measurements of $a_{\Lambda}$ favor a negative value for ${ }_{\Lambda}^{12} \mathrm{C}$ and a positive value for ${ }_{\Lambda}^{5} \mathrm{He}$ [5-9]. On the other hand, from the pioneering work of Block and Dalitz [10] there have been many theoretical attempts dedicated to solving the puzzle. The earliest studies were based on the simplest model of the virtual pion exchange [11]. This model naturally explains the long-range part of the two-body interaction, and it reproduces reasonably well the total decay rate but fails badly in reproducing the other observables. In order to achieve a better description, some improvements have been introduced: (i) models that include the exchange of different combinations of other heavier mesons, such as $\eta, K, \rho, \omega$ and $K^{*}$ [12-19] to reproduce the short-range part of the interaction; (ii) analysis of the two-nucleon $(2 N)$ stimulated process $\Lambda N N \rightarrow N N N$ [20,21]; (iii) inclusion of interaction

*Electronic address: barbero@venus.fisica.unlp.edu.ar terms that violate the isospin $\Delta T=1 / 2$ rule [22-24]; (iv) description of the short-range baryon-baryon interaction in terms of quark degrees of freedom [25,26]; and (v) correlated (in the form of $\sigma$ and $\rho$ mesons) and uncorrelated two-pion exchanges [27-30]. We emphasize that none of these models gives a fully satisfactory description of all the NMHWD observables simultaneously, even though a consistent (though not sufficient) increase of $\Gamma_{n / p}$ has been found. So far, only Jun [24] has been able to reproduce well the $\Gamma_{\mathrm{NM}}$ and $\Gamma_{n / p}$ (but not $a_{\Lambda}$ ) data by employing, in addition to the one-pion exchange, an entirely phenomenological four-baryon point interaction for the short-range part, including the $\Delta T=3 / 2$ contribution, and fixing the model coupling constants. Concerning the proton asymmetry parameter $a_{\Lambda}$, all existing calculations based on strict one-meson exchange models [14-16,18,19,26,31,32] find values between -0.73 and -0.19 for ${ }_{\Lambda}^{5} \mathrm{He}[33,34]$ and when results are available in the same model, very similar values for ${ }_{\Lambda}^{12} \mathrm{C}$. The final state interactions effect, although it attenuates the $a_{\Lambda}$ value, does not reverse the sign of the parameter [34]. Only two recent theoretical calculations show some agreement with the experimental data: (i) a first application of effective field theory (EFT) to non-mesonic decay [35] and (ii) a very recent extension of the direct-quark interaction model, which strongly violates the $\Delta T=1 / 2$ rule, to include $\sigma$ meson exchange [30]. However, in both cases, a nuclear matter formalism (which does not include the full contribution of transitions coming from nucleon states beyond the $s$ shell) is used and could give, therefore, a limited description for $p$-shell hypernuclei or, even worse, for heavier ones.

The EFT approach from Ref. [35] suggests that in order to reproduce the NMHWD data, the microscopic models should be supplemented by isospin and spin-independent central interactions. Motivated by this fact and to set a more detailed description than obtained from previous nuclear matter calculations [30], we analyze the influence of the scalar-isoscalar $\sigma$ meson exchange over the NMHWD. We will work within the shell model (SM) formalism developed in Refs. [17-19], which explicitly includes the contribution of transitions originated from states beyond the $s$ shell. The $\sigma$ meson will be added to the standard strangeness-changing weak $\Lambda N \rightarrow$ $N N$ transition potential already including the exchange of 
TABLE I. PC and PV constants for neutron and proton NMHWD rates of ${ }_{\Lambda}^{5} \mathrm{He}$ obtained with $\Lambda_{\sigma}=1.2 \mathrm{GeV}$ [30]. Parenthetically we give the results with $\Lambda_{\sigma}=1.5 \mathrm{GeV}$.

\begin{tabular}{lcc}
\hline \hline$\mu_{\sigma}(\mathrm{MeV})$ & 550 & 750 \\
\hline$a_{n}^{\mathrm{PC}}$ & $0.0096(0.0126)$ & $0.0010(0.0018)$ \\
$b_{n}^{\mathrm{PC}}$ & $1.0806(0.9459)$ & $3.4141(2.4946)$ \\
$c_{n}^{\mathrm{PC}}$ & $0.0000(0.0000)$ & $0.0000(0.0000)$ \\
$a_{n}^{\mathrm{PV}}$ & $0.0022(0.0031)$ & $0.0003(0.0005)$ \\
$b_{n}^{\mathrm{PV}}$ & $7.3380(6.1721)$ & $21.0396(14.6053)$ \\
$c_{n}^{\mathrm{PV}}$ & $0.0508(0.0510)$ & $0.0509(0.0509)$ \\
$a_{p}^{\mathrm{PC}}$ & $0.0192(0.0251)$ & $0.0019(0.0036)$ \\
$b_{p}^{\mathrm{PC}}$ & $1.3623(1.1915)$ & $4.2979(3.1322)$ \\
$c_{p}^{\mathrm{PC}}$ & $0.1422(0.1421)$ & $0.1421(0.1422)$ \\
$a_{p}^{\mathrm{PV}}$ & $0.0014(0.0020)$ & $0.0002(0.0004)$ \\
$b_{p}^{\mathrm{PV}}$ & $12.4826(10.5148)$ & $35.8000(24.8719)$ \\
$c_{p}^{\mathrm{PV}}$ & $0.1398(0.1398)$ & $0.1399(0.1397)$ \\
\hline \hline
\end{tabular}

the complete pseudoscalar and vector mesons octet $\left(\pi, \eta, K, \rho, \omega, K^{*}\right)$. This model will be referenced as OCT $+\mathrm{S}$, to differentiate it from our previous $\pi+\eta+K+\rho+\omega+K^{*}$ model, designated as OCT.

For the pseudoscalar and vector mesons octet, the weak $(W)$ and a strong $(S)$ vertices in the $\Lambda N \rightarrow N N$ decay will be described by means of the same interaction Hamiltonians given in Ref. [15] and adopted in Refs. [17-19]. This leads to the OCT exchange potential

$$
V^{\mathrm{OCT}}(\mathbf{r})=\sum_{i=\pi, \eta, K, \rho, \omega, K^{*}} \bar{V}_{i}^{(0)}(\mathbf{r})
$$

with $\bar{V}_{i}^{(0)}(\mathbf{r})$ defined in Ref. [18]. In this equation, we neglected all kinematic and first-order nonlocality corrections because, as extensively discussed in Ref. [18], although their effects can be very important for particular transitions, they do not affect the main decay observables very much. For the $\sigma$ meson, we assume weak and strong coupling Hamiltonians of the form [Ref. [30], Eq. (17)]

$$
\begin{aligned}
& \mathcal{H}_{\Lambda N \sigma}^{W}=G_{F} \mu_{\pi}^{2} \bar{\psi}_{N}\left(A_{\sigma}+B_{\sigma} \gamma_{5}\right) \phi_{\sigma}\left(\begin{array}{l}
0 \\
1
\end{array}\right) \psi_{\Lambda}, \\
& \mathcal{H}_{N N \sigma}^{S}=g_{N N \sigma} \bar{\psi}_{N} \phi_{\sigma} \psi_{N},
\end{aligned}
$$

where $\psi_{N}$ and $\psi_{\Lambda}$ are the baryon fields, $\phi_{\sigma}$ is the meson field, and $A_{\sigma}$ and $B_{\sigma}$ are the weak parity-conserving (PC) and parity-violating (PV) coupling constants, respectively, which will be considered as adjustable parameters of the model. For the strong coupling constant, we will assume the value $g_{N N \sigma} \simeq g_{N N \pi}=13.3$. The resulting nonrelativistic one $\sigma$ exchange potential is

$\bar{V}_{\sigma}^{(0)}(\mathbf{r})=G_{F} \mu_{\pi}^{2} g_{N N \sigma}\left[A_{\sigma} f_{C}\left(r, \mu_{\sigma}\right)-i \frac{B_{\sigma}}{2 \bar{M}} f_{V}\left(r, \mu_{\sigma}\right), \sigma_{1} \cdot \hat{\mathbf{r}}\right]$,

where $\mu_{\sigma}$ is the $\sigma$ meson mass, $\bar{M}=\left(M_{\Lambda}+M\right) / 2$ with $M$ and $M_{\Lambda}$ being the nucleon and $\Lambda$ masses, respectively, and all the remaining notation has the same meaning as in Ref. [18]. Thus, we have

$$
V^{\mathrm{OCT}+\mathrm{S}}(\mathbf{r})=V^{\mathrm{OCT}}(\mathbf{r})+\bar{V}_{\sigma}^{(0)}(\mathbf{r}) .
$$

Using the SM formalism developed in Refs. [17,18], we evaluate the transition rates by means of Fermi's golden rule: $\Gamma_{i}=4 \pi \sum_{M_{I}} \sigma\left(J_{I} M_{I} ;-1 / 2, m_{t_{i}}\right)\left(i=n, p, m_{t_{n}}=-1 / 2\right.$, $\left.m_{t_{p}}=1 / 2\right)$, where

$$
\begin{aligned}
\sigma\left(J_{I} M_{I} ; m_{t_{1}}, m_{t_{2}}\right)= & \int d \Omega_{p_{1}} \int d F \sum_{s_{1} s_{2} J_{F} M_{F}} \mid\left\langle\mathbf{p}_{1} s_{1} t_{1} \mathbf{p}_{2}\right. \\
& \left.\times s_{2} t_{2} J_{F} M_{F}|V| J_{I} M_{I}\right\rangle\left.\right|^{2},
\end{aligned}
$$

with $\int d F \cdots=2 \pi \int \frac{p_{1}^{2} d p_{1}}{(2 \pi)^{3}} \frac{p_{2}^{2} d p_{2}}{(2 \pi)^{3}} \delta$ (E.C.) $\cdots$. The initial and final nuclear states are described in the extreme $p$ - $h$ model as excitations over the core, i.e. $\left|J_{I}\right\rangle \rightarrow\left|j_{\Lambda}\right\rangle$ and $\left|J_{F}\right\rangle \rightarrow\left|j_{b}^{-1}\right\rangle$ for ${ }_{\Lambda}^{5} \mathrm{He}$ and $\left|J_{I}\right\rangle \rightarrow\left|j_{\Lambda} j_{a}^{-1} ; J_{I}\right\rangle$ and $\left|J_{F}\right\rangle \rightarrow\left|j_{a}^{-1} j_{b}^{-1} ; J_{F}\right\rangle$ for ${ }_{\Lambda}^{12} \mathrm{C}$. The finite nucleon size (FNS) effects are phenomenologically implemented by a monopole form factor $\left(\Lambda_{M}^{2}-\mu_{M}^{2}\right) /$ $\left(\Lambda_{M}^{2}+q^{2}\right)$, with $\Lambda_{M}$ the cutoff for the meson $M$ [17]. The initial and final short-range correlations (SRC) are simulated, respectively, by means of the correlation functions $[17,18]$

$$
\begin{aligned}
& g_{\Lambda N}(r)=\left(1-e^{-r^{2} / \alpha^{2}}\right)^{2}+\beta r^{2} e^{-r^{2} / \gamma^{2}}, \\
& g_{N N}(r)=1-j_{0}\left(q_{c} r\right),
\end{aligned}
$$

with $\alpha=0.5 \mathrm{fm}, \beta=0.25 \mathrm{fm}^{-2}, \gamma=1.28 \mathrm{fm}$, and $q_{c}=$ $3.93 \mathrm{fm}^{-1}$. The partial rates can be parametrized as

$$
\Gamma_{i}=a_{i}^{\mathrm{PC}}\left(A_{\sigma}-b_{i}^{\mathrm{PC}}\right)^{2}+c_{i}^{\mathrm{PC}}+a_{i}^{\mathrm{PV}}\left(B_{\sigma}-b_{i}^{\mathrm{PV}}\right)^{2}+c_{i}^{\mathrm{PV}}
$$

TABLE II. Experimental data for the NMHWD of ${ }_{\Lambda}^{5} \mathrm{He}$ and ${ }_{\Lambda}^{12} \mathrm{C}$ (total width is in units of $\Gamma_{0}=2.50 \times 10^{-6} \mathrm{eV}$ ).

\begin{tabular}{lcc}
\hline \hline Observable & ${ }_{\Lambda}^{5} \mathrm{He}$ & ${ }_{\Lambda}^{12} \mathrm{C}$ \\
\hline$\Gamma_{\mathrm{NM}}$ & $0.424 \pm 0.024[6]$ & $0.940 \pm 0.035[6]$ \\
& $0.41 \pm 0.14[36]$ & $1.14 \pm 0.2[36]$ \\
$\Gamma_{n / p}$ & $0.39 \pm 0.11[4]$ & - \\
& $(0.45-0.51) \pm 0.15[5]$ & $0.87 \pm 0.09 \pm 0.21[5]$ \\
& $0.45 \pm 0.11 \pm 0.03[6]$ & $0.56 \pm 0.12 \pm 0.04[6]$ \\
$a_{\Lambda}$ & $0.93 \pm 0.55[36]$ & $1.33_{-0.81}^{+0.12[36]}$ \\
& $0.07 \pm 0.08_{-0.00}^{+0.08}[5]$ & $-0.24 \pm 0.26_{-0.00}^{+0.08}[5]$ \\
& $0.11 \pm 0.08 \pm 0.04[6,7]$ & $-0.20 \pm 0.26 \pm 0.04[6,7]$ \\
\hline \hline
\end{tabular}


TABLE III. Adjusted $\sigma$ meson coupling constants.

\begin{tabular}{lcrrrr}
\hline \hline$\mu_{\sigma}(\mathrm{MeV})$ & & 550 & 550 & 750 & \multicolumn{1}{c}{750} \\
$\Lambda_{\sigma}(\mathrm{GeV})$ & & 1.2 & 1.5 & 1.2 & 1.5 \\
Sol I & $A_{\sigma}$ & 0.67 & 0.57 & 2.12 & 1.53 \\
& $B_{\sigma}$ & 13.39 & 11.18 & 38.39 & 26.64 \\
\multirow{3}{*}{ Sol II } & $A_{\sigma}$ & 2.08 & 1.83 & 6.56 & 4.74 \\
& $B_{\sigma}$ & 13.08 & 10.92 & 37.50 & 26.57 \\
\hline \hline
\end{tabular}

with coefficients $a_{i}^{X}, b_{i}^{X}$, and $c_{i}^{X}$ containing the SM information. They are listed in Table I for ${ }_{\Lambda}^{5} \mathrm{He}$ and for different $\mu_{\sigma}$ values. We have observed from the numerical evaluation of the coefficients that they are roughly insensible to the particular correlation function used, but dependent on the $\Lambda_{\sigma}$ value. We include in Table I the results for two different values of this cutoff.

Solving the equation system obtained by fixing $\Gamma_{n}+\Gamma_{p}$ and $\Gamma_{n} / \Gamma_{p}$ to the ${ }_{\Lambda}^{5} \mathrm{He}$ experimental central values from Ref. [6] (shown in Table II), we obtain two sets of solutions for $A_{\sigma}$ and $B_{\sigma}$, exhibited in Table III for two different values of $\mu_{\sigma}$ and $\Lambda_{\sigma}$.

We have found that if FNS and SRC are eliminated at all, there are no single solutions for $A_{\sigma}$ and $B_{\sigma}$ that make the theoretical results consistent with the central values of the experiments. This clearly shows the relevance of these two effects over the parameters. It is important to note that the experimental data have large error bars, and as a consequence, the parameters can vary within a finite region, as shown in Fig. 1 for a particular value of $\mu_{\sigma}$ and $\Lambda_{\sigma}$.

In the following, we evaluate $a_{\Lambda}$ using the SM formalism recently developed in Ref. [19]:

$$
a_{\Lambda}=\frac{12 \pi f}{\left(J_{I}+1\right) \Gamma_{p}} \sum_{M_{I}} M_{I} \sigma\left(J_{I} M_{I} ;-1 / 2,1 / 2\right),
$$

( $f=1$ for ${ }_{\Lambda}^{5} \mathrm{He}$ and $f=-2$ for ${ }_{\Lambda}^{12} \mathrm{C}$ ), leading to

$$
a_{\Lambda}=\left(k_{1} A_{\sigma} B_{\sigma}+k_{2} A_{\sigma}+k_{3} B_{\sigma}+k_{4}\right) / \Gamma_{p},
$$

where the constants $k_{i}$ (containing the SM information) will be given below for a particular value of $\mu_{\sigma}$ and $\Lambda_{\sigma}$. Numerical results obtained for ${ }_{\Lambda}^{5} \mathrm{He}$ with the central fixed values of the

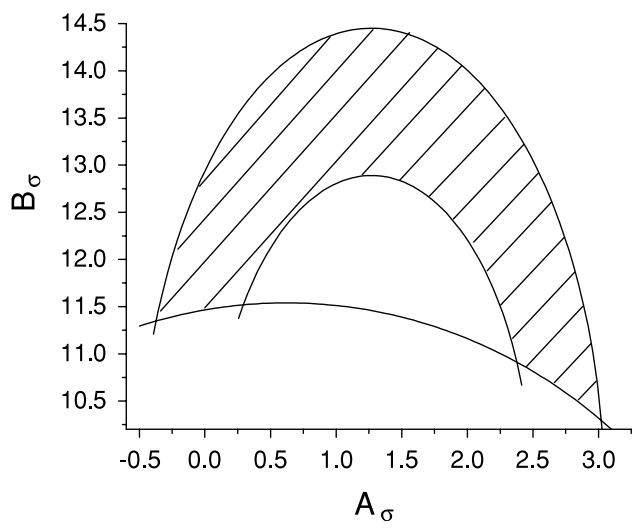

FIG. 1. Allowed region for the $\sigma$ meson coupling constants for $\mu_{\sigma}=550 \mathrm{MeV}$ and $\Lambda_{\sigma}=1.2 \mathrm{GeV}$.
TABLE IV. Predicted hypernuclear weak decay observables for different sets of $\sigma$ coupling constants, mass, and cutoff ( $\Gamma_{\mathrm{NM}}$ is given in units of $\Gamma_{0}=2.50 \times 10^{-6} \mathrm{eV}$ ).

\begin{tabular}{lccccccc}
\hline \hline$\mu_{\sigma}$ & $\Lambda_{\sigma}$ & \multirow{2}{*}{$A_{\sigma}$} & $B_{\sigma}$ & ${ }_{\Lambda}^{5} \mathrm{He}$ & \multicolumn{3}{c}{${ }_{\Lambda}^{12} \mathrm{C}$} \\
\cline { 5 - 8 }$(\mathrm{MeV})$ & $(\mathrm{GeV})$ & & & $a_{\Lambda}$ & $\Gamma_{\mathrm{NM}}$ & $\Gamma_{n / p}$ & $a_{\Lambda}$ \\
\hline- & - & 0 & 0 & -0.507 & 1.166 & 0.267 & -0.508 \\
550 & 1.2 & 0.67 & 13.39 & -0.326 & 0.776 & 0.427 & -0.322 \\
550 & 1.2 & 2.08 & 13.08 & -0.362 & 0.793 & 0.435 & -0.348 \\
550 & 1.5 & 0.57 & 11.18 & -0.328 & 0.769 & 0.415 & -0.326 \\
750 & 1.2 & 2.12 & 38.39 & -0.326 & 0.767 & 0.416 & -0.324 \\
750 & 1.2 & 6.56 & 37.50 & -0.362 & 0.780 & 0.423 & -0.342 \\
750 & 1.5 & 1.53 & 26.64 & -0.325 & 0.765 & 0.411 & -0.325 \\
\hline \hline
\end{tabular}

weak coupling constants have been collected in Table IV, together with the predicted values of all the observables for the $p$-shell ${ }_{\Lambda}^{12} \mathrm{C}$ hypernucleus.

They clearly show that our results are not sensible to the particular $\mu_{\sigma}, \Lambda_{\sigma}, A_{\sigma}$, and $B_{\sigma}$ set selected, because in all cases our OCT $+\mathrm{S}$ model predicts the values $a_{\Lambda}\left({ }_{\Lambda}^{5} \mathrm{He}\right) \simeq$ $-0.34, \Gamma_{\mathrm{NM}}\left({ }_{\Lambda}^{12} \mathrm{C}\right) \simeq 0.78, \Gamma_{n / p}\left({ }_{\Lambda}^{12} \mathrm{C}\right) \simeq 0.43$ and $a_{\Lambda}\left({ }_{\Lambda}^{12} \mathrm{C}\right) \simeq$ -0.33 . This gives evidence of the fact that these variables are strongly correlated between them and shows the stability of our results (in spite of the fact that $\mu_{\sigma}$ and $\Lambda_{\sigma}$ are unknown parameters of the theory) [37]. A straightforward comparison of OCT $+\mathrm{S}$ results with the OCT ones shows that the inclusion of the $\sigma$ meson reduces the results for $\Gamma_{\mathrm{NM}}$ by $\sim 40 \%$. In addition, the neutron-to-proton branching ratio $\Gamma_{n / p}$ is increased $\sim 36 \%$. These last two effects occur because the $\sigma$ meson strongly reduces the PV contribution of the proton-induced decay, as shown in Table $\mathrm{V}$ and as can be seen from Eq. (6) for $B_{\sigma} \simeq b_{p}^{\mathrm{PV}}$. Furthermore, $\sigma$ meson helps bring to near zero the asymmetry $a_{\Lambda}$, increasing it $\sim 35 \%$.

To illustrate the $A_{\sigma}$ and $B_{\sigma}$ dependence of the NMHWD observables, we show in Fig. 2 our results for ${ }_{\Lambda}^{5} \mathrm{He}$. We see that within our OCT $+\mathrm{S}$ model, a bigger value for $A_{\sigma}$ and a smaller for $B_{\sigma}$ are necessary for reversing the asymmetry sign. This can be understood by noting that for $\mu_{\sigma}=550 \mathrm{MeV}$ and $\Lambda_{\sigma}=1.2 \mathrm{GeV}$, we have $k_{1}=-0.010, k_{2}=0.127, k_{3}=$ 0.014 , and $k_{4}=-0.275$. These $A_{\sigma}$ and $B_{\sigma}$ values overestimate the total rate $\Gamma_{\mathrm{NM}}$ and strongly underestimate the ratio $\Gamma_{n / p}$. From Figs. 1 and 2, we observe that the best value that can be reached for $a_{\Lambda}\left({ }_{\Lambda}^{5} \mathrm{He}\right)$ within our model, consistent with the experimental data of partial rates, is obtained with $A_{\sigma}=3.1, B_{\sigma}=10.1$, leading to $a_{\Lambda}=-0.24$, which is not consistent with the experimental value. For the $p$-shell ${ }_{\Lambda}^{12} \mathrm{C}$ hypernucleus, we get a better agreement with data, which

TABLE V. Comparison of partial rate contributions within OCT and OCT $+\mathrm{S}$ models for $\mu_{\sigma}=550 \mathrm{MeV}, \Lambda_{\sigma}=1.2 \mathrm{GeV}, A_{\sigma}=0.67$, and $B_{\sigma}=13.39$ (in units of $\Gamma_{0}=2.50 \times 10^{-6} \mathrm{eV}$ ).

\begin{tabular}{lccccc}
\hline \hline & Model & $\Gamma_{n}^{\mathrm{PC}}$ & $\Gamma_{n}^{\mathrm{PV}}$ & $\Gamma_{p}^{\mathrm{PC}}$ & $\Gamma_{p}^{\mathrm{PV}}$ \\
\hline${ }_{\Lambda}^{5} \mathrm{He}$ & $\mathrm{OCT}$ & 0.011 & 0.167 & 0.178 & 0.364 \\
& $\mathrm{OCT}+\mathrm{S}$ & 0.002 & 0.130 & 0.151 & 0.141 \\
${ }_{\Lambda}^{12} \mathrm{C}$ & $\mathrm{OCT}$ & 0.024 & 0.222 & 0.296 & 0.624 \\
& $\mathrm{OCT}+\mathrm{S}$ & 0.005 & 0.227 & 0.251 & 0.293 \\
\hline \hline
\end{tabular}




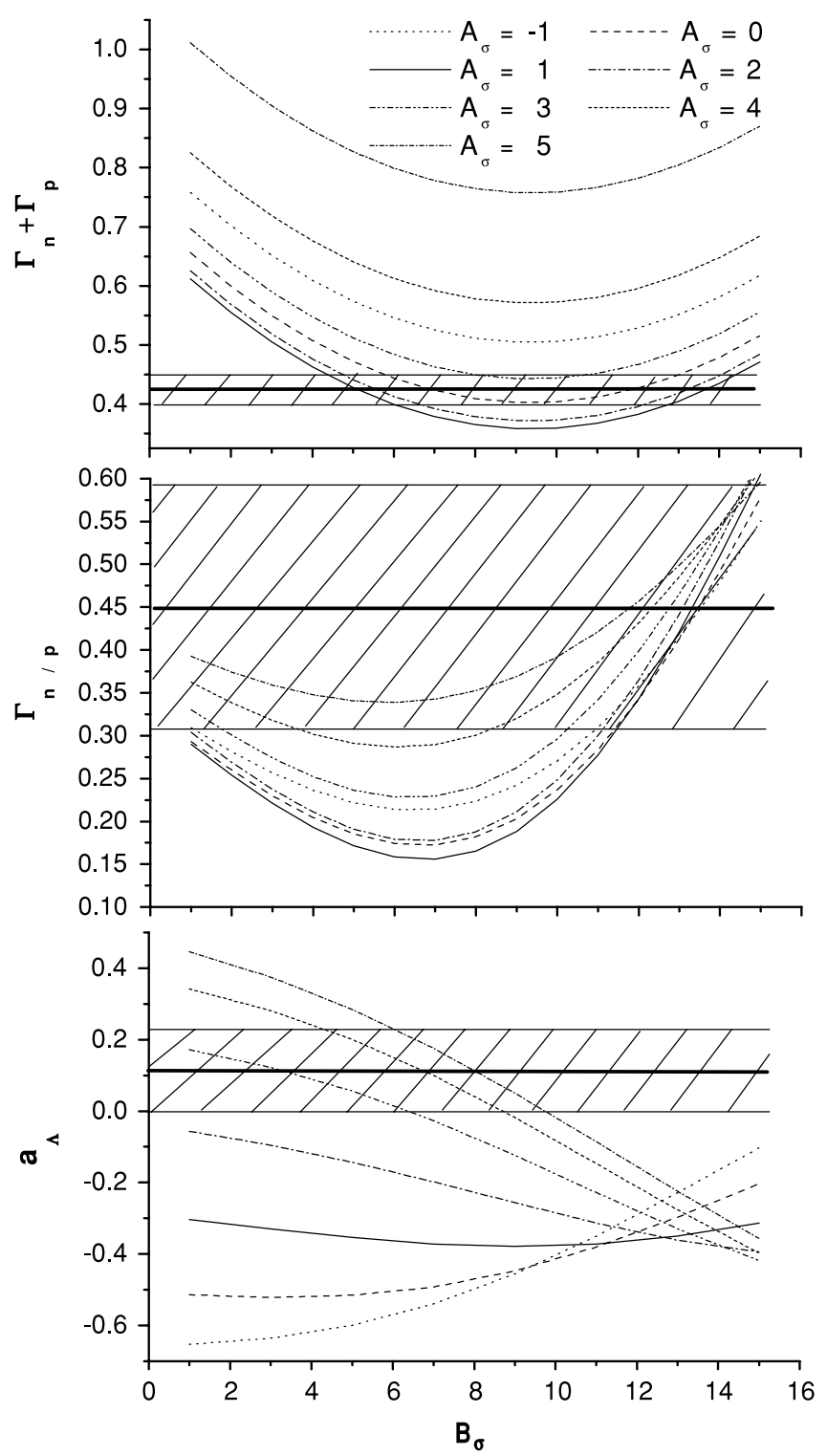

FIG. 2. $A_{\sigma}$ and $B_{\sigma}$ dependence of the ${ }_{\Lambda}^{5} \mathrm{He}$ decay observables, for $\mu_{\sigma}=550 \mathrm{MeV}$ and $\Lambda_{\sigma}=1.2 \mathrm{GeV}$. Shaded region stands for experimental values [6] with error bars.

have large error bars. Thus, the OCT $+\mathrm{S}$ model implemented with the SM formalism shows that the inclusion of the $\sigma$ meson is not enough to reproduce simultaneously all the data, especially the positive proton asymmetry value for ${ }_{\Lambda}^{5} \mathrm{He}$. At this point, it is important to note that we have not included here the contribution of the $2 N$ induced decay to $\Gamma_{\mathrm{NM}}$. We only qualitatively discuss the effect of this process on our results, since there is not a SM formalism for $2 N$ decay in the literature. We will adopt $\Gamma_{2 N} \simeq 0.2\left(\Gamma_{n}+\Gamma_{p}\right)$, as calculated within nuclear matter inspired models $[4,6,38]$. This means that we must adjust $A_{\sigma}$ and $B_{\sigma}$ in $\Gamma_{n}+\Gamma_{p}$ to reproduce $\left(\Gamma_{\mathrm{NM}}\right)_{\exp } / 1.2 \sim 0.35$. (The data for $\Gamma_{n / p}=0.45$ is not modified because it has been obtained by a coincidence method independent of the final state interactions and $2 N$ mechanism [6].) Therefore, from Fig. 2 it is clear that no single values for parameters $A_{\sigma}$ and $B_{\sigma}$ could reproduce these values for $\Gamma_{n}+\Gamma_{p}$ and $\Gamma_{n / p}$. Nevertheless, as the $\sigma$ inclusion has reduced the contribution of $\Gamma_{n}+\Gamma_{p}$ by $\sim 40 \%$ from the old OCT model, and as the relation $\Gamma_{2 N}=0.2\left(\Gamma_{n}+\Gamma_{p}\right)$ is only valid for a nuclear matter calculation, a consistent evaluation of $\Gamma_{2 N}$ within the SM with the $\sigma$ inclusion could produce a different $\Gamma_{2 N} /\left(\Gamma_{n}+\Gamma_{p}\right)$ ratio; but our conclusion is not definitive.

Summarizing, we have analyzed the $\sigma$ meson contribution to the NMHWD observables in the framework of a SM formalism [17-19], which carefully includes the effect of the $p$-shell state decays, both in the partial decay rates and in the asymmetry parameter evaluation. From our results, we conclude that even though the $\sigma$ meson contributes to reducing the total decay width $\Gamma_{\mathrm{NM}}$ and increasing the neutron-to-proton branching ratio $\Gamma_{n / p}$, it is not enough to reproduce the more recent experimental data on the asymmetry. Nevertheless, it improves sensibly the values of $a_{\Lambda}$ for the $s$-shell ${ }_{\Lambda}^{5} \mathrm{He}$; and for the $p$-shell ${ }_{\Lambda}^{12} \mathrm{C}$ hypernucleus, we have obtained a better agreement. Our results also corroborate the theoretical expectation that $a_{\Lambda}$ should have only a moderate dependence on the particular hypernucleus considered, as can be seen from Table IV. We suggest three possible alternatives for future improvements of the model: (i) additional degrees of freedom should be added to our SM formalism to improve the description of the experimental data, for example, $\Delta I=3 / 2$ contributions in the same footing of Ref. [30] (but now in a finite nucleus scheme) where a better agreement has been obtained for ${ }_{\Lambda}^{5} \mathrm{He}$; (ii) a different decay mechanism should be introduced for $s$ - and $p$-shell hypernuclei, beyond the Born approximation; (iii) the $2 \mathrm{~N}$ weak decay contribution should be included within a SM formalism. Finally, we have clearly shown that the effects of an isoscalar central spin-independent interaction included through the $\sigma$ meson would not be enough to reproduce simultaneously all the NMHWD observables, without additional changes in the nuclear model and/or the decay mechanism.

\section{ACKNOWLEDGMENT}

C. B. thanks CONICET (Argentina) for support under Grant No. 666 and M. N. Barbero for a careful reading of the manuscript.
[1] H. Outa et al., KEK Report No. KEK-PS E462, 2000.

[2] A. Feliciello, Nucl. Phys. A691, 170 (2001); P. Gionatti, ibid. A691, 483 (2001).

[3] R. L. Gill, Nucl. Phys. A691, 180 (2001).

[4] G. Garbarino, A. Parreño, and A. Ramos, Phys. Rev. C 69, 054603 (2004); Nucl. Phys. A754, 137 (2005).
[5] H. Bhang et al., Nucl. Phys. A754, 144 (2005).

[6] H. Outa et al., Nucl. Phys. A754, 157 (2005).

[7] T. Maruta et al., Nucl. Phys. A754, 168 (2005).

[8] S. Ajimura et al., Phys. Lett. B282, 293 (1992).

[9] S. Ajimura et al., Phys. Rev. Lett. 84, 4052 (2000).

[10] M. M. Block and Dalitz, Phys. Rev. Lett. 11, 96 (1963). 
[11] J. B. Adams, Phys. Rev. 156, 1611 (1967) (Cf. correction pointed out in Ref. [12].)

[12] B. H. J. McKellar and B. F. Gibson, Phys. Rev. C 30, 322 (1984).

[13] K. Takeuchi, H. Takaki, and H. Bandō, Prog. Theor. Phys. 73, 841 (1985).

[14] J. F. Dubach, G. B. Feldman, B. R. Holstein, and L. de la Torre, Ann. Phys. (NY) 249, 146 (1996).

[15] A. Parreño, A. Ramos, and C. Bennhold, Phys. Rev. C 56, 339 (1997) and references therein.

[16] A. Parreño and A. Ramos, Phys. Rev. C 65, 015204 (2001).

[17] C. Barbero, D. Horvat, F. Krmpotić, T. T. S. Kuo, Z. Narancić, and D. Tadić, Phys. Rev. C 66, 055209 (2002).

[18] C. Barbero, C. de Conti, A. P. Galeao, and F. Krmpotić, Nucl. Phys. A726, 267 (2003).

[19] C. Barbero, A. P. Galeao, and F. Krmpotić, Phys. Rev. C 72, 035210 (2005).

[20] A. Ramos, E. Oset, and L. L. Salcedo, Phys. Rev. C 50, 2314 (1994).

[21] A. Ramos, M. J. Vicente-Vacas, and E. Oset, Phys. Rev. C 55, 735 (1997); 66, 039903(E) (2002).

[22] A. Parreño, A. Ramos, C. Bennhold, and K. Maltman, Phys. Lett. B435, 1 (1998).

[23] W. M. Alberico and G. Garbarino, Phys. Lett. B486, 362 (2000).

[24] J.-H. Jun, Phys. Rev. C 63, 044012 (2001).

[25] T. Inoue, M. Oka, T. Motoba, and K. Itonaga, Nucl. Phys. A633, 312 (1998).
[26] K. Sasaki, T. Inoue, and M. Oka, Nucl. Phys. A669, 331 (2000); A678, 455(E) (2000).

[27] M. Shmatikov, Phys. Lett. B322, 311 (1994); Nucl. Phys. A580, 538 (1994).

[28] K. Itonaga, T. Ueda, and T. Motoba, Nucl. Phys. A577, 301 (1994); A585, 331 (1995); A639, 329 (1998).

[29] E. Jido, E. Oset, and J. A. Palomar, Nucl. Phys. A694, 525 (2001).

[30] K. Sasaki, M. Izaki, and M. Oka, Phys. Rev. C 71, 035502 (2005).

[31] K. Itonaga, T. Ueda, and T. Motoba, Phys. Rev. C 65, 034617 (2002); K. Itonaga, T. Motoba, and T. Ueda, in Electrophoto Production of Strangeness on Nuand Nuclei (Sendai03), edited by K. Maeda, H. Tamura, S. N. Nakamura, and O. Hashimoto (World Scientific, Singapore, 2004), p. 397.

[32] K. Sasaki, T. Inoue, and M. Oka, Nucl. Phys. A702, 477 (2002).

[33] W. M. Alberico and G. Garbarino, Phys. Rep. 369, 1 (2002).

[34] W. M. Alberico, G. Garbarino, A. Parreño, and A. Ramos, Phys. Rev. Lett. 94, 082501 (2005).

[35] A. Parreño, C. Bennhold, and B. R. Holstein, Phys. Rev. C 70, 051601(R) (2004) .

[36] J. J. Szymanski et al., Phys. Rev. C 43, 849 (1991).

[37] G. López Castro and A. Mariano, Nucl. Phys. A697, 440 (2001).

[38] W. M. Alberico, A. De Pace, G. Garbarino, and A. Ramos, Phys. Rev. C 61, 044314 (2000). 